PROCEEDINGS OF THE

AMERICAN MATHEMATICAL SOCIETY

Volume 139, Number 2, February 2011, Pages 687-689

S 0002-9939(2010)10549-X

Article electronically published on August 24, 2010

\title{
CLOSED GEODESICS AND VOLUME GROWTH OF RIEMANNIAN MANIFOLDS
}

\author{
JIANMING WAN
}

(Communicated by Jianguo Cao)

\begin{abstract}
In this paper, we study the relation between the existence of closed geodesics and the volume growth of open Riemannian manifolds with nonnegative curvature.
\end{abstract}

\section{INTRODUCTION}

Let $M^{n}$ be an $n$-dimensional complete, noncompact Riemannian manifold with sectional curvature $K_{M} \geq 0$. Let

$$
\alpha_{M}=\lim _{r \rightarrow \infty} \frac{\operatorname{Vol}(B(p, r))}{\omega_{n} r^{n}},
$$

where $\operatorname{Vol}(B(p, r))$ is the volume of geodesic ball in $M^{n}$ with radius $r$ around $p$ and $\omega_{n}$ denotes the volume of unit ball in $R^{n}$. From [6] we know that $\alpha_{M}$ is independent of the choice of base point $p$. By the Bishop-Gromov volume comparison theorem, we have $0 \leq \alpha_{M} \leq 1$ and $M^{n}$ is isometric to $R^{n}$ if and only if $\alpha_{M}=1$.

The main goal of this paper is to prove the following theorem.

Theorem 1.1. Let $M^{n}$ be a complete noncompact manifold with nonnegative section curvature. If $M^{n}$ contains a closed geodesic, then the volume growth $\alpha_{M}=0$. In other words, if $\alpha_{M}>0$, then $M^{n}$ does not contain any closed geodesic.

We may look at Theorem 1.1 in an intuitive manner: To an open manifold with nonnegative section curvature, the closed geodesic will make the manifold shrink.

By the Cheeger-Gromoll soul theorem (see [2]), if the soul of $M^{n}$ is not a point, then $M^{n}$ must contain at least one closed geodesic. If the soul is one point, $M^{n}$ still may have many closed geodesics. The following is a simple example.

Example 1.2. Let $M^{2}=C_{+} \cup S_{1}^{2}$ be a cylinder $C_{+}=S^{1} \times[0, \infty)=\left\{(x, y, z) \mid x^{2}+\right.$ $\left.y^{2}=1, z \geq 0\right\}$ glued to the lower hemisphere $S_{-}^{2}=\left\{(x, y, z) \mid x^{2}+y^{2}+z^{2}=1, z \leq 0\right\}$. Then the soul of $M^{2}$ is a point, but $M^{2}$ admits infinitely many closed geodesics.

In fact, our theorem is more significant when the soul is one point. In this case, the volume growth gives a sufficient condition of the nonexistence of closed geodesics, which is not a trivial thing.

Received by the editors February 25, 2010 and, in revised form, April 17, 2010.

2010 Mathematics Subject Classification. Primary 53C20; Secondary 53C22.

Key words and phrases. Closed geodesics, volume growth.

(C)2010 American Mathematical Society Reverts to public domain 28 years from publication 
Remark 1.3. In what follows, we always assume that manifolds are complete noncompact with nonnegative sectional curvature.

\section{Proof of Theorem 1.1}

The proof of Theorem 1.1 is based on the following two lemmas.

Lemma 2.1. Let $\sigma(t)$ be a closed geodesic of $M^{n}$ with canonical parameter of the arc such that $\sigma(0)=\sigma(b)=p, \sigma^{\prime}(0)=\sigma^{\prime}(b)$, where $b$ is the length of $\sigma(t)$. For any ray $\gamma(t)$ starting at $p$, we have $\alpha=\angle\left(\sigma^{\prime}(0), \gamma^{\prime}(0)\right)=\pi / 2$.

Proof. Let $l$ be the length of $\sigma(t)$ from $\sigma(0)$ to $\sigma(l)$. By the Toponogov comparison theorem [1, we have

$$
t^{2}+l^{2}-2 t l \cos \alpha \geq d^{2}(\sigma(l), \gamma(t)) .
$$

Thus

$$
\cos \alpha \leq \frac{t^{2}+l^{2}-d^{2}(\sigma(l), \gamma(t))}{2 t l},
$$

where $d(.,$.$) is the distance function. Recalling the condition of the Toponogov$ comparison theorem [1], one only needs $l<\infty$. Let $l=b$. Then $t=d(\sigma(b), \gamma(t))$. Thus

Let $t \longrightarrow \infty$. Then

$$
\cos \alpha \leq \frac{b}{2 t} .
$$

$$
\cos \alpha \leq 0,
$$

so

$$
\alpha \geq \pi / 2 \text {. }
$$

Considering $\sigma(b-t)$, we obtain

$$
\pi-\alpha \geq \pi / 2
$$

Hence

$$
\alpha=\pi / 2 .
$$

Remark 2.2. Lemma 2.1 can also be deduced by analytic methods. For example, see Theorem 1.10 of [2. But our proof is more direct.

The next lemma is due to Ordway, Stephens and Yang [6]. It shows that $\alpha_{M}$ is determined by "the volume of rays".

Lemma 2.3. Let $\Sigma=\left\{\nu \in S_{p} M \mid \exp _{p}(t \nu)\right.$ is a ray, $\left.t \geq 0\right\}$. Here $S_{p} M$ is the unit sphere in $T_{p} M$. Set

$$
C(\Sigma)=\left\{q \in M \mid q=\exp _{p}(t \nu), \nu \in \Sigma, t \geq 0\right\}
$$

and

Then we have

$$
B(\Sigma, r)=B(p, r) \cap C(\Sigma) .
$$

$$
\alpha_{M}=\lim _{r \rightarrow \infty} \frac{\operatorname{Vol}(B(\Sigma, r))}{\omega_{n} r^{n}} .
$$

The proof of Lemma 2.3 is based on the Bishop-Gromov volume comparison theorem. For details, one may see [6].

Now we can prove Theorem 1.1. 
Proof. If $M^{n}$ contains a closed geodesic, by Lemma 2.1, we have mes $(\Sigma)=0$ (induced measure of the unit sphere). By Fubini's theorem, for any $r>0$ we have

$$
\operatorname{mes}\left(\exp ^{-1}(B(\Sigma, r))\right)=0 \text {. }
$$

Since $\exp$ is $C^{\infty}$, by Sard's theorem [3], for any $r>0$ we have

$$
\operatorname{Vol}(B(\Sigma, r))=0 \text {. }
$$

Then by Lemma 2.3 , we have $\alpha_{M}=0$.

\section{An Application of Theorem 1.1}

Combining with the Cheeger-Gromoll soul theorem (see 2]), we get another proof of Marenich and Toponogov's beautiful theorem (see [5]):

Theorem 3.1. If $\alpha_{M}>0$, then $M^{n}$ is diffeomorphic to $R^{n}$.

Proof. If $M^{n}$ is not diffeomorphic to $R^{n}$, by the Cheeger-Gromoll soul theorem, the soul (a totally geodesic submanifold) of $M^{n}$ is not a point. Then the soul must contain a closed geodesic (since any compact manifold contains at least one closed geodesic [4]). It is also the closed geodesic of $M^{n}$, which is a contradiction to Theorem 1.1.

Remark 3.2. By a different method, Theorem 3.1 is also a consequence of Perelman's celebrated flat strip theorem (cf. [7]).

\section{ACKNOWLEDGMENT}

The author would like to thank the referee for helpful suggestions.

\section{REFERENCES}

1. J. Cheeger and D. Ebin, Comparison Theorems in Riemannian Geometry. North-Holland, Amsterdam, 1975. MR0458335 (56:16538)

2. J. Cheeger and D. Gromoll, The structure of complete manifolds of nonnegative curvature, Ann. of Math. (2) 96, 413-443, 1972. MR0309010 (46:8121)

3. M. W. Hirsch, Differential Topology. Springer-Verlag, 1976. MR 0448362 (56:6669)

4. W. Klingenberg, Riemannian Geometry. de Gruyter, 1982. MR666697(84j:53001)

5. V. B. Marenich and V. A. Toponogov, Open manifolds of nonnegative Ricci curvature with rapidly growing volume (Russian). Sib. Mat. Zh. 26, no. 4 (152), 191-194, 1985. MR0804031 (86k:53061)

6. D. Ordway, B. Stephens and D. G. Yang, Large volume growth and finite topological type, Proceedings of the A.M.S. 128, no. 4, 1191-1196, 2000. MR.1705745(2000i:53059)

7. G. Perelman, Proof of the soul conjecture of Cheeger and Gromoll. J. Differential Geom. 40, no. 1, 209-212, 1994. MR.1285534 (95d:53037)

Center of Mathematical Sciences, Zhejiang University, Hangzhou, Zhejiang, 310027, People's Republic of China

E-mail address: wanj_m@yahoo.com.cn 